\title{
Management of Concomitant Abdominal Aortic Aneurysm and Intra-abdominal, Retroperitoneal Malignancy
}

\author{
VLADISLAV TRESKA ${ }^{1}$, JIRI MOLACEK ${ }^{1}$, BOHUSLAV CERTIK ${ }^{1}$, KAREL HOUDEK ${ }^{1}$, PETR HOSEK ${ }^{2}$, \\ VERONIKA SOUKUPOVA ${ }^{1}$, CHRISTIANA STOGEROVA $^{1}$ and ANETA SVEJDOVA ${ }^{1}$ \\ ${ }^{1}$ Department of Surgery, School of Medicine in Pilsen, Charles University in Prague, Prague, Prague, Czech Republic; \\ ${ }^{2}$ Biomedicine Center, School of Medicine, Pilsen, Czech Republic
}

\begin{abstract}
Background/Aim: As the population ages, there are increasing findings of coincidental diseases such as abdominal aortic aneurysm (AAA) and intra-abdominal, retroperitoneal malignancy. The aim of this study was to propose an optimal treatment procedure for these patients. Patients and Methods: Over a twenty-year-period, surgery was performed on a total of 1,098 patients with AAA and 32 (2.9\%) patients with AAA and intra-abdominal, retroperitoneal malignancy: 18 renal, 6 colorectal carcinomas, 3 carcinomas of the small intestine, 3 primary liver tumours, 1 stomach carcinoma and 1 teratoma. The median age of patients was 72.5 years, there were 20 men (62.5\%) and 12 women (37.5\%). A one-stage procedure was performed on 19 patients (59.4\%), and a two-stage procedure on 13 (40.6\%) patients. Results: The average time of hospitalization was $12.4 \pm 6.9$ days (median=11.0 days) for one-stage procedure, for a two-stage procedure $21.3 \pm 9.3$ days (median $=20.0$ days), $p=0.0045$. Seven patients $(21.9 \%$ ) died within 30 days after the operation. All the deaths were in the group of one-stage procedures $(p=0.0252)$. The 1-, 3- and 5year overall survival for patients following one-stage and twostage procedures was 61.0/56.3/51.5\% and 89.0/79.9/53.0\% respectively ( $p=0.1199)$. Conclusion: Symptomatic disease must be resolved first. Two-stage procedures are the method of choice and offer better short-term results compared to onestage procedures.
\end{abstract}

As the population ages, and with the use of precise radiodiagnostics during the examination of patients, there are

This article is freely accessible online.

Correspondence to: Vladislav Treska, MD, Ph.D., Professor of Surgery, Head of the Department of Surgery, University Hospital, School of Medicine, Pilsen, Czech Republic. Tel: +420 3771042701 , e-mail: treska@fnplzen.cz

Key Words: Abdominal aortic aneurysm, intra-abdominal, retroperitoneal malignancy, surgical procedures, long-term results. increasing accidental discoveries of coincidental diseases, such as abdominal aortic aneurysm (AAA) with intra- and retroperitoneal malignancy (IRM). Depending on the type of malignancy, the concurrent incidence of both diseases ranges from 0.5 to $13.4 \%(1,2)$. The aim of the study was to propose the optimal treatment procedure on the basis of an evaluation of the short- and long-term results of surgical treatment for concurrent incidence of AAA and IRM.

\section{Patients and Methods}

The Ethics committee approval for this study was received from the Institutional Ethics Committee. The study was performed in accordance with the ethical standards laid down in the 1964 Declaration of Helsinki. We obtained written informed consent from all patients who participated in this study. In the period from $12 / 1999$ to $12 / 2019$ operations were performed on a total of 1098 patients with AAA having an average age of $71.2 \pm 7.9$ years (median $=71.7$ years). There were 912 men $(83.1 \%)$, and 186 women $(16.9 \%)$. The diameter of AAA was $61 \pm 22 \mathrm{~mm}$ (median=58 mm). The indications for urgent operation were AAA rupture (rAAA), symptomatic AAA (sAAA), and for elective operations they were asymptomatic AAA (aAAA) with diameter of $\geq 50 \mathrm{~mm}$, or growing AAA where the diameter had increased by $\geq 5 \mathrm{~mm}$ over the past 6 months. $670(61.0 \%)$ of patients had aAAA, 207 (18.9\%) sAAA without rupture and $221(20.1 \%)$ with rAAA. We performed AAA resection with replacement using tubular or bifurcated prosthesis for 899 patients $(81.9 \%)$, and an endovascular aneurysm repair- EVAR - was performed in 199 patients (18.1\%).

Over the same period, jointly with AAA, 32 patients (2.9\%) were diagnosed with IRM. There were 20 men (62.5\%), and 12 women $(37.5 \%)$. The average age of patients was $72.0 \pm 6.9$ years (median=72.5). The average age of patients for one-stage and twostage procedures was $71.2 \pm 7.0$ years (median 72.3 years) and $73.1 \pm 6.8$ (median 74.6 years) respectively. The diameter of AAA was $67 \pm 19 \mathrm{~mm}$ (median $66 \mathrm{~mm}$ ). In the case of one-stage procedures the diameter of AAA was $65 \pm 19 \mathrm{~mm}$ (median=60 $\mathrm{mm}$ ), for two-stage procedures $70 \pm 18 \mathrm{~mm}$ (median $=67 \mathrm{~mm})-p=0.3909$. There were 18 renal, 6 colorectal carcinomas, 3 carcinomas of the small intestine, 3 primary liver carcinomas, 1 stomach carcinoma and 1 retroperitoneal teratoma. Twenty-six AAA were asymptomatic $(81.3 \%)$, two were sAAA $(6.3 \%)$ and there were four rAAA $(12.5 \%)$. AAA resection was performed in $30(93.8 \%)$ and EVAR 
Table I. Patient characteristics (12/1999-12/2019).

\begin{tabular}{|c|c|c|c|}
\hline & $\mathrm{N}=1,098(\%)$ & $\mathrm{N}=32(\%)$ & $p$-Value \\
\hline Age (years) & $71.2 \pm 7.9($ median $=71.7)$ & $72.0 \pm 6.9($ median $=72.5)$ & 0.5946 \\
\hline Male & $912(83.1)$ & $20(62.5)$ & 0.0026 \\
\hline Female & $186(16.9)$ & $12(37.5)$ & \\
\hline $\mathrm{AAA}(\mathrm{mm})$ & $61 \pm 22($ median $=58)$ & $67 \pm 19($ median $=66)$ & 0.0547 \\
\hline $\mathrm{aAAA}$ & $670(61.0)$ & $26(81.3)$ & 0.0612 \\
\hline SAAA & $207(18.9)$ & $2(6.3)$ & \\
\hline rAAA & $221(20.1)$ & $4(12.5)$ & \\
\hline Resection & $899(81.9)$ & $30(93.8)$ & 0.1003 \\
\hline EVAR & $199(18.1)$ & $2(6.3)$ & \\
\hline
\end{tabular}

AAA: Abdominal aortic aneurysm; aAAA: asymptomatic AAA; sAAA: symptomatic AAA; rAAA: rupture AAA; EVAR: endovascular aneurysm repair.

Table II. Group of patients with intra-abdominal, retroperitoneal malignancy ( $N=32)$.

\begin{tabular}{lccc}
\hline & One-stage procedure $(\%)$ & Two-stage procedure $(\%)$ & $p$-Value \\
\hline $\mathrm{N}$ & $19(59.4)$ & $13(40.6)$ & 0.3771 \\
Male & $11(57.9)$ & $9(69.2)$ & 0.7128 \\
Female & $8(42.1)$ & $4(30.8)$ & 0.4513 \\
Age (year) & $71.2 \pm 7.0$ (median=72.3) & $73.1 \pm 6.8($ median=74.6) & 0.3909 \\
AAA (mm) & $65 \pm 19$ (median=60) & $70 \pm 18$ mm (median=67) & 1 \\
Resection & $18(94.7)$ & $12(92.3)$ & 0.0199 \\
EVAR & $1(5.3)$ & $9(69.2)$ & $0(0)$ \\
aAAA & $17(89.5)$ & $4(30.8)$ & \\
SAAA & $2(10.5)$ & $0(0)$ & \\
rAAA & &
\end{tabular}

AAA: Abdominal aortic aneurysm; aAAA: asymptomatic AAA; sAAA: symptomatic AAA; rAAA: rupture AAA; EVAR: endovascular aneurysm repair.

in $2(6.3 \%)$. A one-stage procedure was performed on 19 patients $(59.4 \%)$, and a two-stage procedure on $13(40.6 \%)$.

The indication for a one-stage procedure was AAA resection (usually with tubular replacement) with simple operation for tumour (such as resection of small intestine, small liver operation, nephrectomy, right hemicolectomy, transverse colon resection) or uncomplicated EVAR with concurrent simple operation due to malignancy. In one-stage procedures with resection of intestine and open operation for AAA, first of all AAA was dealt with, often with covering of retroperitoneum with omentum, draping with antiseptic drapes and subsequent operation on bowel. A laparoscopic operation for IRM (nephrectomy, bowel resection) was performed on three patients $(15.8 \%)$, EVAR on one patient with aAAA $(5.3 \%)$.

A two-stage procedure was indicated in $13(40.6 \%)$ patients where one of the diseases required technically more demanding surgery, such as large liver resection, open AAA resection with bifurcation reconstruction, AAA resection due to rupture etc. Minimally invasive laparoscopic surgery (such as nephrectomy, partial nephrectomy, intestinal resection) was performed on six $(46.2 \%)$ of patients and EVAR on one (7.7\%) of patients (Tables I and II).

Standard frequency tables and descriptive statistics were used to characterize the patient sample. Because of its asymmetrical and significantly non-normal distribution (as reviewed in histogram and using Shapiro-Wilk test), the duration of hospitalization was analyzed using Mann-Whitney $U$-test. Associations between categorical variables were analyzed by crosstabulation with Chisquared or Fisher exact test. Overall survival (OS) was determined from the date of AAA surgery to the date of death, regardless of its cause. Patients who had not died were censored at the date of last follow-up. Observed proportions surviving at given time points (1, 3,5 years) were calculated from the Kaplan-Meier estimates of survival functions using linear interpolation between the nearest complete observations. Median follow-up of 41.2 months was estimated from OS data using the inverse Kaplan-Meier method. Significance of observed differences in OS was tested using GehanWilcoxon test. All reported $p$-values are two-tailed and the level of statistical significance was set at $\alpha=0.05$. Statistical processing and testing was performed in STATISTICA data analysis software system (StatSoft, Inc.2013, Version 12, www.statsoft.com).

\section{Results}

The average time of hospitalization for all groups of patients $(\mathrm{N}=1,098)$ following operations for aAAA was $11.3 \pm 7.9$ days (median=9.0 days), the number after resections for aAAA and EVAR was $12.2 \pm 8.6$ (median $=10$ days), and 8.6 \pm 4.6 (median $=8$ days, $p=0.0001$ ) respectively. In the case of 


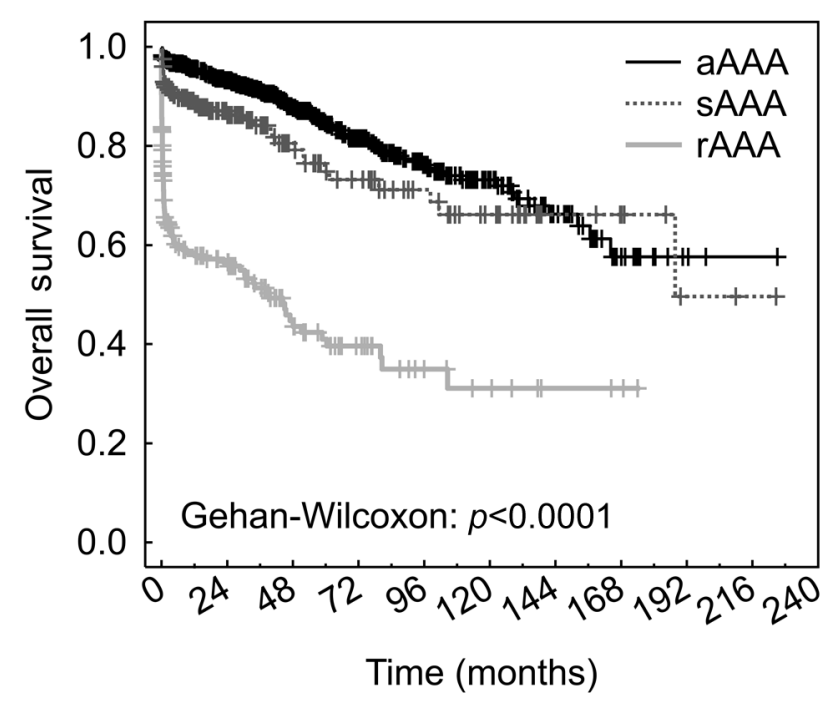

Figure 1. Overall survival of patients according to AAA symptomatology $(N=1,098)$.

resections for sAAA and rAAA the average hospitalization period was $13.9 \pm 8.7$ days (median $=11.0$ days) and 14.6 \pm 13.4 days (median $=13.0$ days), respectively ( $p=0.4574$ ). Out of 670 patients, 16 died (2.4\%) within 30 days after an open operation for aAAA, and one out of $199(0.5 \%)$ died after EVAR $(p=0.0864)$. The 30 -day mortality of resection for sAAA and rAAA was $14(6.8 \%)$ and $67(30.3 \%)$, respectively $(p=0.0001)$. Clavien-Dindo grade III-IV post-operative complications occurred in $23(3.4 \%)$ patients following resection and two $(1.0 \%)$ after EVAR $(p=0.5055)$ for aAAA, in $18(8.7 \%)$ patients after resection for sAAA and 37 (16.7\%) for rAAA ( $p=0.0293$ ). The overall 1-, 3- and 5-year survival for the entire group of patients was $87.2,82.3$ and $74.5 \%$ respectively, of which for aAAA $95.5 / 91.1 / 84.3 \%$, sAAA $88.5 / 83.7 / 74.5 \%$ and rAAA it was $57.9 / 50.9 / 40.0 \%$ (Figure 1).

In the group of 32 patients with a malignancy, the average hospitalization time for one-stage procedures was 12.4 \pm 6.9 days (median=11.0 days) and for two-stage procedures $21.3 \pm 9.3$ days (median $=20.0$ days),$p=0.0045$. Seven patients $(21.9 \%)$ died within 30 days after one-stage procedure, no one after two-stage procedure $(p=0.0252)$. In the case of seven $(36.8 \%)$ patients following a one-stage procedure a revision operation was required as a result of serious Clavien-Dindo grade III-IV complications (in the case of six patients after an open operation, and in the case of one patient following EVAR), in the case of two-stage procedures we had three $(23.1 \%)$ serious complications that we managed to resolve ( $p=0.4673)$, Table III. The $1-, 3$ - and 5 -year overall survival for patients following one-stage and two-stage procedures was $61.0 / 56.3 / 51.5 \%$ and $89.0 / 79.9 / 53.0 \%$ respectively $(p=0.1199$ ) (Figure 2 ). Long-
Table III. Results of surgical treatment of patients with AAA and AAA with concomitant intra - abdominal, retroperitoneal malignancy.

\begin{tabular}{|c|c|c|}
\hline \multicolumn{2}{|l|}{$\mathrm{N}=1098(\%)$} & $p$-Value \\
\hline \multicolumn{3}{|l|}{ Time of hospitalization } \\
\hline aAAA - resection & $12.2 \pm 8.6$ (median $=10$ days $)$ & \\
\hline aAAA - EVAR & $8.6 \pm 4.6$ (median=8 days) & 0.0001 \\
\hline sAAA - resection & $13.9 \pm 8.7($ median $=11$ days $)$ & \\
\hline rAAA - resection & $14.6 \pm 13.4$ (median=13 days) & 0.4574 \\
\hline \multicolumn{3}{|l|}{ Mortality } \\
\hline aAAA - resection & $16(2.4)$ & \\
\hline aAAA - EVAR & $1(0.5)$ & 0.0864 \\
\hline sAAA - resection & $14(6.8)$ & \\
\hline rAAA - resection & $67(30.3)$ & 0.0001 \\
\hline \multicolumn{3}{|c|}{ Morbidity (Clavien - Dindo III-IV) } \\
\hline aAAA - resection & $23(3.4)$ & \\
\hline aAAA - EVAR & $2(1.0)$ & 0.5055 \\
\hline sAAA - resection & $18(8.7)$ & \\
\hline rAAA - resecton & $37(16.7)$ & 0.0293 \\
\hline $\mathrm{N}=32$ & & $p$-Value \\
\hline \multicolumn{3}{|l|}{ Time of hospitalization } \\
\hline One - stage (days) & $12.4 \pm 6.9($ median $=11)$ & \\
\hline Two - stage & $21.3 \pm 9.3($ median $=20)$ & 0.0045 \\
\hline \multicolumn{3}{|l|}{ Mortality } \\
\hline One - stage & $7(21.9 \%)$ & \\
\hline Two - stage & $0(0 \%)$ & 0.0252 \\
\hline \multicolumn{3}{|c|}{ Morbidity (Clavien - Dindo III-IV) } \\
\hline One - stage & $7(36.8 \%)$ & \\
\hline Two - stage & $3(23.1 \%)$ & \\
\hline 0.4673 & & \\
\hline
\end{tabular}

AAA: Abdominal aortic aneurysm; aAAA: asymptomatic AAA; sAAA: symptomatic AAA; rAAA: rupture AAA; EVAR: endovascular aneurysm repair.

term results in patients with combined procedures compared to operations for AAA only, are given in Figure 3.

\section{Discussion}

As the number of older inhabitants within the population increases, so does the incidence of AAA and IRM. Many etiopathogenic factors are common to both groups of patients. These include age above 65 years, male gender, smoking, high cholesterol level and genetic factors. Most often AAA occurs jointly with colorectal $(0.5-4.9 \%)$ carcinoma, renal carcinoma (1.3-4.2\%), stomach $(2-3.8 \%)$ carcinoma and hepatocellular carcinoma $(0.4-0.8 \%)(3,4)$. The coincidence of both diseases represents a treatment challenge for the surgeon who has to decide on a one-stage or two-stage procedure, an open AAA resection or EVAR, and on dealing with IRM through an open or laparoscopic (robotic) operation. For IRM the type of tumour and perioperative oncological treatment are taken into the treatment consideration. 


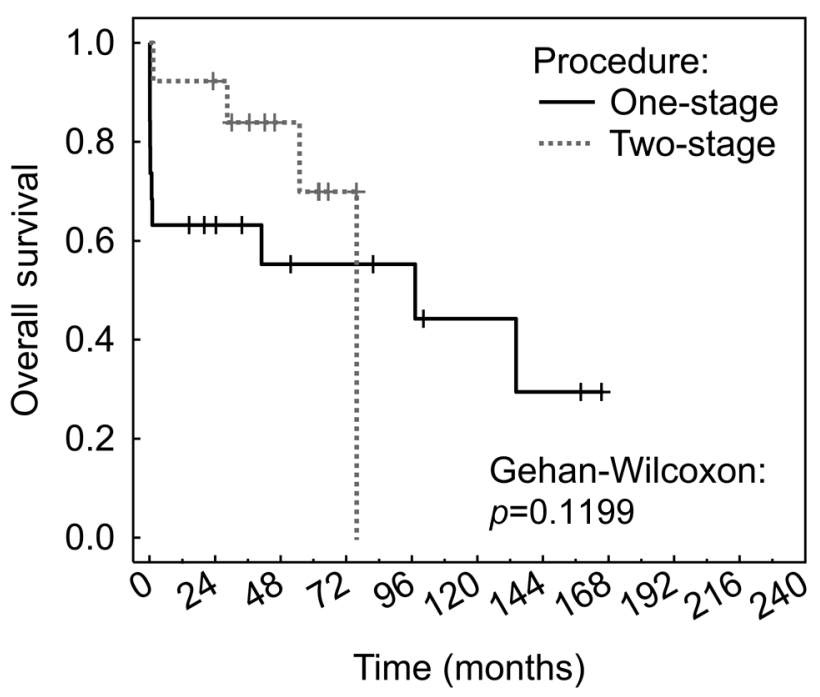

Figure 2. Overall survival of patients after one- and two-stage procedure $(N=32)$.

Those who favour a one-stage procedure solution (5-7) see the benefit of this procedure in the early resolution of both nosological units without the undue delay of a two-stage procedure. There is, thus, no danger of an AAA rupture if the IRM is dealt with first during a two-stage procedure. It is stated that $6-10 \%$ of AAA rupture during the first three weeks after another intra-abdominal operation (in particular if there are complications) as a result of the start of collageno- and elastolysis in the case of activation of metalloproteinase, inflammatory cytokines, deterioration in nutrition of patients in post-operative period and when adjuvant chemotherapy is started. Most often it involves an AAA with a diameter greater than $60 \mathrm{~mm}$ (8). In contrast with this, if the IRM is dealt with in the second stage, and complications arise after the first AAA operation, the interval between operations may be extended incommensurately and there may occur progress with possible generalisation of IRM. These are primarily tumours in higher TNM stage (9). Further possible advantages of an one-stage procedure include resolution of both diseases during a single operation, under general anaesthesia only once.

Those who oppose one-stage procedures $(10,11)$ are concerned with the risk of infection of the vascular prosthesis, in particular during the resection of colorectal tumours concurrently with open AAA resection due to infectious content in the large intestine. This complication is more at the theoretical level, because current perioperative procedures (preparation of bowel, antibiotic prophylaxis) combined with adequate surgical and anaesthesiological techniques preclude these complications in practice $(12,13)$. They also state that one-stage procedures are more time consuming, with higher

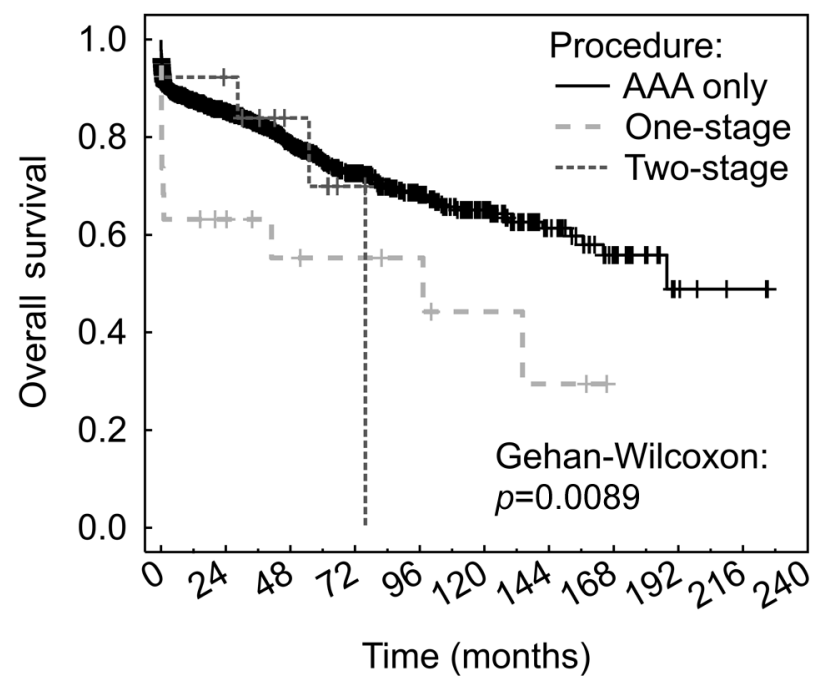

Figure 3. Comparison of overall survival of the whole group of patients after AAA surgery only $(N=1,066)$ and patients with one-or two-stage procedures $(N=32)$.

operation stress for the patient and weakened immunity, which plays a role in the long-term results of tumour treatment. So, one-stage procedures are burdened by a higher incidence of post-operative complications.

Both schools of thought concur in the indication for acute (urgent) operations for symptomatic diseases with AAA (symptomatology, rupture), or with IRM (bowel obstruction, bleeding, perforation), where the symptomatic disease is dealt with first, and then the secondary disease after the patient has healed (14).

From the aspect of operational technique for AAA or IRM, open, endovascular and mini-invasive procedures (laparoscopy, robotic surgery) are possible. An undoubted advantage of EVAR given good anatomical conditions of AAA is the minimally invasive nature of the procedure, less surgical stress, lower number of complications (especially cardiac, pulmonary and renal), possibility of performing intervention under analgesia-sedation, significant reduction in need for blood replacement, faster convalescence and shorter hospitalization period. Another advantage of EVAR during a one-stage procedure is minimisation of infection of the endograft compared to open AAA resection. In two-stage procedures it is usually possible to deal with IRM in a short 2-5 day interval after EVAR, and there is no danger of needless delay in oncological treatment. One unambiguous advantage is the use of EVAR during one stage with an IRM operation, because in most cases it represents a low operational burden for the patient. The advantage of EVAR is its use where an open resection would be very complicated, as is the case in patients with a "hostile abdomen" $(15,16)$. 
One certain problem is the higher incidence of reinterventions for EVAR (up to $13 \%$ ), primarily as a result of thrombosis, leaks and migration of endovascular prosthesis $(17,18)$. Thrombogenicity is a paraneoplastic manifestation and one of the complications of oncological treatment, and the lithotomy position of the patient during operations on the pelvis is a risk factor for endograft thrombosis. The incidence of abdominal angina after EVAR ranges in general from 1.5 to $3 \%$. For patients with a tumour on the left side of the colon and rectum, during an EVAR it is important to preserve the patency of at least one internal iliac artery during exclusion of the inferior mesenteric artery for preservation of collateral circulation for this part of the intestine, which is essential for resections and the vitality of anastomoses in this area $(19,20)$. If for various reasons this is not possible, it is better to choose an open operation, primarily for AAA with aneurysms in the iliac arteries with implantation of inferior mesenteric artery to preserve the vitality of bowel anastomosis in this area. This procedure can also be recommended for patients following a prior resection of colorectal cancer on the left colon and rectum with patent inferior mesenteric artery (21).

Some studies also warn of possible worse incorporation of EVAR for patients with adjuvant oncological treatment which leads to reduced synthesis of collagen, elastin and function of smooth muscle tunica media, increased activity of metalloproteinase, release of inflammatory cytokines and inhibition of DNA synthesis (22-24). The problem of oncological treatment combined with an AAA operation arises primarily for more advanced tumours of the rectum with a need for neoadjuvant chemoradiotherapy. So, for these patients it is a good idea to use an AAA solution through EVAR during the first stage. It is also better to perform surgery associated with an operation on a rectal carcinoma in two stages, with surgery for AAA in the first stage as a result of possible infection complications given an insufficiency of anastomosis or wound infection, which could significantly delay dealing with the AAA operation $(25,26)$.

An open AAA resection with replacement using bifurcated or tubular graft is possible in both procedures, i.e., during one-stage or two-stage procedures (27). This surgery also displays minimal mortality and morbidity in the case of elective operations in large centres, even in patients who are anatomically unsuitable for EVAR. At our facility we use infection-resistant vascular prostheses in these cases. However, the post-operative convalescence of patients, and thus also hospitalization, is longer, and there is a much more frequent need for post-operative monitoring of patients at the intensive care unit. If we select the two-stage surgery, we have to assume an approximately two-week interval between operations necessary for the convalescence of the patient.

One-stage open procedure can be used well for patients with renal carcinoma and patients with tumours of the small and large intestine (in particular on the ascending and transverse colon) and liver (28). For bowel surgery it is recommended that the AAA resection first be performed, and then after coverage of the vascular prosthesis with AAA wall, rear sheet of retroperitoneum (sometimes even omentum) and draping with anti-septic drapes, perform bowel resection with minimisation of possible infection of vascular reconstruction. Prophylactic administration of antibiotics is a matter of course. In the group of our patients we observed no infections of the vascular prosthesis when this approach was applied. It is advantageous to use retroperitoneal access for AAA resections (29) in two-stage procedures. It is advantageous as a secondary operation in the case of preceding laparotomies, where we avoid intraabdominal adhesions, or in the first stage, where we thus leave the abdominal cavity free for subsequent open or minimally invasive surgery for IRM.

There is a general increase in the use of minimally invasive laparoscopic approaches in surgery on the gastrointestinal tract and in urology for operations on renal carcinomas, and they can be used well primarily for two-stage surgery, where they are the first operation, and with regard to the rapid convalescence of the patient it is possible to perform AAA resection or EVAR after a short interval of approximately one week. One highly advantageous procedure is a one-stage procedure using both minimally invasive techniques - EVAR with laparoscopic (or robotic) IRM operation (30), which can be performed primarily for anatomically suitable AAA for EVAR and simple laparoscopic interventions.

In addition to the aforementioned, it is necessary to mention the adequacy of using EVAR and open AAA resection in combined operations from the aspect of life expectancy and the economic aspects of operations. Should it involve a patient of high biological age with generalized malignancy and short life prognosis, there is no indication to burden the patient with any operations for AAA or IRM unless absolutely essential. In the case of advanced colorectal carcinomas with bowel obstruction, we choose to insert a bowel stent or perform a palliative operation on the bowel without dealing with AAA. From the aspect of treatment strategy, the multidisciplinary team decision and wishes of the patient have a fundamental role.

We are aware of the many limitations of this study. It is a retrospective, non-randomised study with limited number of patients. However, in recent literature dealing with the same matter there are also small, heterogeneous retrospective cohorts, often based on case series, with absent perioperative data. In terms of selection of patients our work is also of a heterogeneous nature, primarily from the aspect of malignant diseases, although this was unavoidable given the low level of representation of individual tumours. Moreover, as a result of the limitation of the patient cohort it is not possible to comment on the advantages or disadvantages of a given 
operation technique (open or minimally invasive). In our overall cohort of patients with AAA there is an evident very low proportion of EVAR compared with open operations, which is given by the greater preference for open operations in general at our Department, primarily in the first years of the twenty-year period under examination. We still believe that open AAA resection is better in the cases of concomitant IRM for its evaluation and choice of optimal onco-surgical procedure. But over the past decade there has been a gradual increase in the proportion of EVAR at the expense of open AAA resections in patients with AAA only. We did not evaluate the reason for worse long-term results in patients with combined procedures compared to operations for AAA only. They are most likely due to a shorter life expectancy in patients with intra- or retroperitoneal malignancy. However, a larger group of patients with combined procedures and a more targeted analysis of the deaths of both monitored groups of patients would be necessary for a precise specification of the causes.

\section{Conclusion}

A multidisciplinary team and the wishes of the patient decide on the indication of type and means of operation. The selected method should be tailored to the given patient. It is always necessary to consider the risk of the given surgery in contrast with disease-related mortality. Symptomatic, lifethreatening disease must be resolved without delay in the first stage. Based on our results we recommend performance of two-stage procedures, which have better short-term postoperative results. From the aspect of technique of both operations, it is better to select minimally invasive operations, which represent lower operating stress for the patient and have shorter time of hospitalization.

\section{Conflicts of Interest}

The Authors report no conflicts of interest.

\section{Authors' Contributions}

Treska Vladislav: Study design, operation procedures, writing of article. Molacek Jiri: Operation procedures, study design. Certik Boihuslav: Operation procedures, data collection. Houdek Karel: operation procedures, data collection. Hosek Petr: Statistical analysis. Soukupova Veronika: data collection, preparation of material for statistical analysis. Stogerova Christiana: data collection, preparation of material for statistical analysis. Svejdova Aneta: data collection, preparation of material for statistical analysis.

\section{Acknowledgements}

This study was supported by Research Project PROGRES Q-39, UNCE/MED/006 Charles University - Center of clinical and experimental liver surgery.

\section{References}

1 Kenyon J, Liu W and Dalgleish A: Report of objective clinical responses of cancer patients to pharmaceutical-grade synthetic cannabidiol. Anticancer Res 38: 5831-5835, 2018. PMID: 30275207. DOI: 10.21873/anticanres.12924

2 Onohara T, Kyuragi R, Inoue K, Yoshida S, Matsumoto T and Furuyama T: Late-onset malignant neoplasms and their prognostic factors after abdominal aortic aneurysm repair. Ann Vasc Surg 56: 194-201, 2019. PMID: 30476610. DOI: 10.1016/j.avsg.2018.08.095

3 Oliveiar-Pinto J, Mosquera N, Vidoedo J, Moreira-Sampaio S and Teixeira J: Co-existence of abdominal aortic aneurysm with urologic neoplasm: Which should be treated first in the endovascular era? Rev Port Cir Cardiotorac Vasc 24: 187-192, 2017. PMID: 29701416.

4 Peeters B, Moreels N, Vermassen F and Herzeele van I: Management of abdominal aortic aneurysm and concomitant malignant disease. J Cardiovasc Surg (Torino) 60: 468-475, 2019. PMID: 30916530. DOI: 10.23736/S0021-9509.19.10946-9

5 Treska V, Hora M, Certik B, Molacek J, Houdek K, Nahlik J and Stransky P: Simultaneous procedure in patients with abdominal aortic aneurysm and renal carcinoma. Rozhl Chir 93: 424-427, 2014. PMID: 25230387.

6 Illuminati G, Pizzardi G, Pasqua R, Calio F G, Chakfe N and Ricco $\mathrm{J}-\mathrm{B}$ : Endovascular exclusion of abdominal aortic aneurysms and simultaneous resection of colorectal cancer. Ann Vasc Surg 58: 16, 2019. PMID: 31009731. DOI: 10.1016/j.avsg.2019.02.006

7 Yubakami M, Hiramatsu K, Yamazaki T, Matsuzaki Y, Houri Y, Watanabe N, Kondo Y, Ujiiie K, Nishimura Y, Todo M, Nakamura Y, Atsuji K, Yamaguchi A and Taniguchi H: The actual state of treatment of gastrointestinal cancer with abdominal aortic aneurysm in our department over the past five years. Gan To Kagaku Ryoho 45: 477-479, 2018. PMID: 29650910.

8 Cvetkovic S, Koncar I, Galun D, Ribac J, Markovic M, Ilic N, Dragas $M$ and Davidovic L: Treatment of a patient with abdominal aortic aneurysm and hepatocellular carcinoma. Ann Vasc Surg 40: 295.e1-295.e4, 2017. PMID: 27890842. DOI: 10.1016/j.avsg.2016.07.075

9 Kumar R, Dattani N, Asaad O, Bown M J, Sayers R D and Saratzis A: Meta-analysis of outcomes following aneurysm repair in patients with synchronous intra-abdominal malignancy. Eur J Vasc Endovasc Surg 52: 747-756, 2016. PMID: 27592036. DOI: $10.1016 /$ j.ejvs.2016.07.084

10 Kouvelos GN, Patelis N, Antoniou GA, Lazaris A, Bali Ch and Matsagkas M: Management of concomitant abdominal aortic aneurysm and colorectal cancer. J Vasc Surg 63: 1384-93, 2016. PMID: 27005754. DOI: 10.1016/j.jvs.2016.01.026

11 Berchiolli R, Mocellin DM, Marconi M, Tomei F, Bargellini I, Zanca R, Erba P and Ferrari M: Ruptured mycotic aneurysm after intravesical instillation for bladder tumor. Ann Vasc Surg 59: 310.e7-310.e11, 2019. PMID: 30802589. DOI: 10.1016/ j.avsg.2018.12.100

12 Amato B, Esposito G, Serra R, Compagna R, Vigliotti G, Bianco T, Massa G, Amato G, Amato M, Massa S and Aprea G: Onestep mini-invasive treatment of abdominal aorto-iliac aneurysm associated with colo-rectal cancer. Int J Surg 12: S193-196, 2014. PMID: 25157990. DOI: 10.1016/j.ijsu.2014.08.343

13 Kawai K., Sunami E., Tanaka J., Tanaka T, Kiyomatsu T, Nozawa H, Kazama S, Kanazawa T, Hosaka A, Ishihara S, Yamaguchi H, Shigematsu K and Watanabe T: Synchronous 
colorectal malignancy and abdominal aortic aneurysm treated with endovascular aneurysm repair followed by laparoscopic colectomy. Int Surg 100: 600-603, 2015. PMID: 25875539. DOI: 10.9738/INTSURG-D-14-00111.1

14 Boyle JR: Management of AAA and concomitant intraabdominal malignancy: the jury is still out. Eur J Vasc Endovasc Surg 52: 757, 2016. PMID: 27756529.

15 Oliveira NFG, Ultee K, van Rijn MJ, Pinto JP, Raa ST, Gonçalves FB, Hoeks SE and Verhagen HJM: Anatomic predictors for late mortality after standard endovascular aneurysm repair. J Vasc Surg 69: 1444-1451, 2019. PMID: 30477942. DOI: $10.1016 /$ j.jvs.2018.07.082

16 Frego M, Lumachi F, Bianchera G, Pilon F, Scarpa M, Ruffolo C, Polese L, Angriman I, Norberto L, Miotto D, Motta R, Zanon A and Picchi G: Risk factors of endoleak following endovascular repair of abdominal aortic aneurysm. A multicentric retrospective study. In Vivo 21: 1099-1102, 2007. PMID: 18210763.

17 Hagihara K, Takahashi H, Miyoshi N, Haraguchi N, Hata T, Matsuda Ch, Mizushima T, Yamamoto H, Doki Y and Mori M: Case of laparoscopic right hemicolectomy for ascending colon cancer after aortic graft replacement and revascularization of the superior mesenteric artery. Asian J Endosc Surg 11: 266-269, 2018. PMID: 29322641. DOI: 10.1111/ases.12461

18 Matsumoto T, Matsuda D, Honma K, Aoyagi Y, Okadome J, Morisaki K, Tanaka S, Saeki H, Oki E and Maehara Y: Onestage procedure for concomitant abdominal aortic aneurysm and gastric cancer. Anticancer Res 35: 6909-12, 2015. PMID: 26637915.

19 Maeda K, Ohki T, Kanaoka Y, Toya N, Baba T, Hara M and Hagiwara S: Current surgical management of abdominal aortic aneurysm with concomitant malignancy in the endovascular era. Surg Today 46: 985-994, 2016. PMID: 26471508. DOI: $10.1007 / \mathrm{s} 00595-015-1262-8$

20 Sugimoto M, Koyama A, Niimi K, Kodama A, Banno H and Komori K: Long-term comparison of endovascular and open repair of abdominal aortic aneurysms: Retrospective analysis of matched cohorts with propensity score. Ann Vasc Surg 43: 96103, 2017. PMID: 28390921. DOI: 10.1016/j.avsg.2017.01.011

21 Quyn AJ, Fraser CG, Rodger J, Digan A, Anderson AS and Steele RJC: Participation in bowel screening among men attending abdominal aortic aneurysm screening. Br J Surg 105: 529-534, 2018. PMID: 29465743. DOI: 10.1002/bjs.10758

22 Blanes Ortí PC, Bernal LR, Requejo García L and Hernandez MM: Abdominal aortic rupture secondary to lymphoma recurrence. Ann Vasc Surg 58: 381, 2019. PMID: 30731218. DOI: $10.1016 /$ j.avsg.2018.10.050
23 Markar SR, Vidal-Diez A, Sounderajah V, Mackenzie H, Hanna GB, Thompson M, Holt P, Lagergren J and Karthikesalingam A: A population-based cohort study examining the risk of abdominal cancer after endovascular abdominal aortic aneurysm repair. J Vasc Surg 69: 1776-1785, 2019. PMID: 30583890. DOI: $10.1016 /$ j.jvs. 2018.09.058

24 Ahmad M, Mistry R, Hodson J and Bradbury AW: How quickly do asymptomatic infrarenal abdominal aortic aneurysms grow and what factors affect aneurysm growth rates? Analysis of a single centre surveillance cohort database. Eur J Vasc Endovasc Surg 54: 597-603, 2017. PMID: 28882515. DOI: 10.1016/j.ejvs. 2017.08 .002

25 Leopardi M, Di Marco E, Musilli A, Ricevuto E, Bruera G and Ventura M: Effects of chemotherapy in petients with concomitant aortic aneurysm and malignant disease. Ann Vasc Surg 45: 268.e13268.e20, 2017. PMID: 28739458. DOI: 10.1016/j.avsg.2017.07.013

26 Mohandas S, Malik HT and Syed I: Concomitant abdominal aortic aneurysm and gastrointestinal malignancy: Evolution of treatment paradigm in the endovascular era - review article. Int J Surg 11: 112-115, 2013. PMID: 23266417. DOI: 10.1016/j.jjsu.2012.11.022

27 Rodd CD and Wolfe J: Complex abdominal aortic aneurysm repair. Acta Chir Belg 106: 458-466, 2006. PMID: 17017709. DOI: $10.1080 / 00015458.2006 .11679932$

28 Hohneck A,Shchetynska-Marinova T, Ruemenapf G, Pancheva M, Hofheinz R, Boda-Heggemann J, Sperk E, Riffel P, Michels J, Borggrefe M, Akin I and Sigl M: Coprevalence and incidence of lung cancer in patients screened for abdominal aortic aneurysm. Anticancer Res 40: 4137-4145, 2020. PMID: 32620663. DOI: 10.21873 /anticanres.14413

29 Lareyre F, Reverso-Meinietti J, Carboni J, Gaudart A, HassenKhodja R and Raffort J: Mycotic aortic aneurysm and infected aortic graft after intravesical bacillus Calmette-Guérin treatment for bladder cancer. Vasc Endovascular Surg 53: 86-91, 2019. PMID: 30213242 . DOI: $10.1177 / 1538574418800128$

30 López Arquillo IM, Vidal Rey J and Encisa de Sá JM: Abdominal aorta aneurysm and synchronous colorectal cancer. Changes in treatment? Cir Esp 95: 114-116, 2017. PMID: 27444265. DOI: 10.1016/j.ciresp.2016.06.003

Received October 14, 2020

Revised October 29, 2020

Accepted November 3, 2020 